\title{
Detection and discrimination of flaws in ball bearings by vibration analysis
}

\author{
Hiroshi Kanai,* Masato Abe,** and Ken'iti Kido* \\ * Research Center for Applied Information Sciences, Tohoku University, \\ Katahira 2-1-1, Sendai, 980 Japan \\ **Education Center for Information Processing, Tohoku University, \\ Kawauchi, Sendai, 980 Japan
}

(Received 25 April 1985)

\begin{abstract}
This paper describes a new automatic method of detecting flaws in ball bearings and classifying them into four categories: normal, flaw on the inner ring, flaw on the outer ring, and flaw on the balls. Detection and classification are currently carried out mainly aurally by inspectors who listen to a vibration signal obtained by a vibration pick-up in an Anderon meter, which is attached to the outer ring of a ball bearing while the inner ring rotates at a uniform speed. There have been some proposals for the automatic detection and classification of flaws which are based on the analysis of the periodicity of vibration pulses excited by flaws. When flaws are slight, however, it is hard to detect them by recently proposed methods because of the following two reasons: (A) the power of the vibration due to flaws is less than that of noise, and (B) the period of flaw pulses is irregular. We have developed a new method, though, by which slight flaws can be detected. We have applied this new method in the flaw detection of small-sized ball bearings: flaws were classified with an accuracy rate of greater than $97 \%$ into the above four categories.
\end{abstract}

PACS number: 43. 85. Ta, 43. 60. Gk

\section{INTRODUCTION}

This paper describes a new diagnostic method for the automatic detection and classification of flaws on the surface of ball bearings. Ball bearings can be classified into the following four categories:

(1) normal,

(2) ones having flaws on the inner rings,

(3) ones having flaws on the outer rings,

(4) ones having flaws on the balls.

When a flawed bearing is used, for example, in a video tape recorder to support the spindle of the head, even flaws of less than $0.01 \mathrm{~mm}$ on the bearing debase the quality of the played-back picture.

Bearings used for such audio/visual purposes are currently checked aurally, one by one, using an Anderon meter ${ }^{1)}$ as follows: a ball bearing is fixed in an Anderon meter and the inner ring is revolved at a uniform speed. The vibration signal is picked up by a vibration pick-up attached to the outer ring. Defects in ball bearings are thus detected and classified aurally by inspectors listening to vibration signals with headphones. However, it requires a great deal of time to train a good inspector. Additionally, the physical and mental condition of an inspector affects the results of detection and classification.

In response, several methods have recently been proposed up to now for the automatic detection and classification of defects in ball bearings. These methods utilize pulses excited when balls encounter flaws on races or when races encounter flaws on balls. Since the rotation speed of the inner ring is known, the periodicity of a vibration signal can be calculated from the shape of the bearing. Hence, defects may be detected and classified by checking whether or not peaks are observed at the expected 
frequencies. Unfortunately, slight flaws cannot be detected by these proposed automatic methods.

Therefore, we have developed a new method for the automatic detection and classification of surface defects in ball bearings, in order to check ball bearings more reliably so as to attain higher standards of stability, and to eliminate tedious labor for workers.

This paper first discusses the reasons why slight flaws cannot be detected by recently proposed automatic methods. Next, a new method is proposed to detect and classify defects even in the case of slight flaws; this method was experimentally tested, the results of which are shared here. It was proved by experiment that defects can be classified into four categories with an accuracy rate of $97.9 \%$.

\section{APPARATUS FOR THE EXPERIMENTS}

Figure 1 shows the block diagram of the experimental system in which a vibration signal is picked up and carried to the laboratory using digital audio tape. The inner ring rotates at a constant speed of 1,800 r.p.m., and the outer one is fixed by axial pressure; the value of the speed and the amount of pressure are the same as those used in practice in video tape recorders. Under such conditions, a flaw causes a radial movement of the outer ring; a signal due to the movement is picked up by a vibration pick-up attached to the outer ring. The signal is amplified

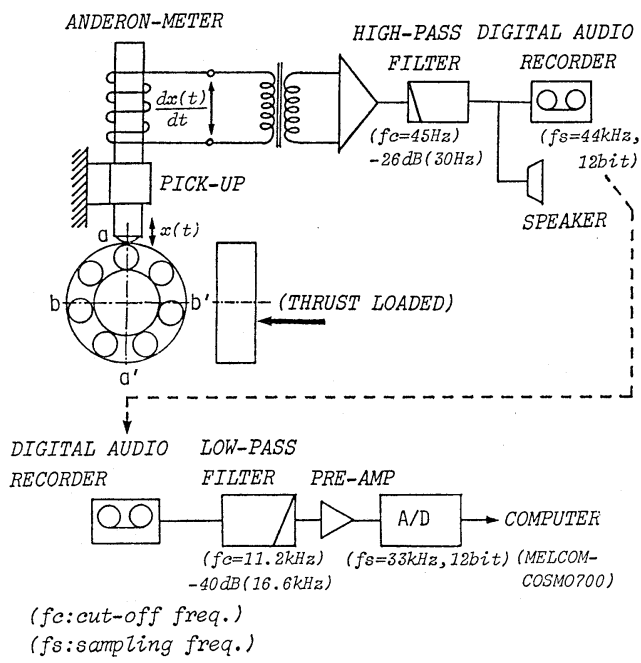

Fig. 1 The block diagram of the procedure for measuring the vibration signal of a ball bearing. and filtered through a high-pass filter to eliminate the primary frequency component $(=30 \mathrm{~Hz})$ corresponding to the rotation of the inner ring. The filtered signal is stored in a high-fidelity digital audio tape. In the laboratory, the signal is played back and is $A / D$ converted with a 12 bits $A / D$ converter at a sampling rate of $30 \mu$ s so as to be processed by a digital computer.

\section{FLAW DETECTION USING THE POWER SPECTRUM OR CEPSTRUM TECHNIQUE}

A vibration signal $x_{1}(n)$ is excited by a flaw on the inner ring, on the outer ring or on the balls. It is assumed in the simplified model that the resonant vibration signal is expressed as the sum of several number of exponentially decaying sinusoidal waves, as follows:

$$
x_{1}(n)=\sum_{i} \exp \left(-\alpha_{i} n\right) \cos \left(\omega_{i} n\right) \cdot u(n),
$$

where $u(n)$ is a unit step function, $\omega_{i}$ is an angular eigen frequency and $\alpha_{i}$ is a damping factor of $i$-th mode. Since the bearing rotates at a uniform speed, the vibration signal $x_{1}(n)$ repeats at a uniform period $T$. The repetition frequency $1 / T$ depends on the position where the flaw is. The frequency is derived theoretically ${ }^{2)}$ using the rolling speed of the inner

Table 1 Equations for the repetition frequencies of flaws and the frequencies calculated for the ball bearing (JIS696).

The number of balls: $\quad Z=7$

Ball diameter: $d=2.7781 \mathrm{~mm}$

Pitch diameter: $D=10.5 \mathrm{~mm}$

Contact angle: $\theta=10.3 \mathrm{deg}$

[at a state with about $1.75 \mathrm{~kg}$ thrust loaded]

(1) Rolling speed of the inner ring:

$f_{1}=30 \mathrm{~Hz}(1,800$ r.p.m. $)$

(2) Rolling speed of the cage (=revolution speed of balls):

$f_{2}=f_{1} \cdot\{1-(d / D) \cos \theta\} / 2=11.2 \mathrm{~Hz}$

(3) Rotating speed of balls:

$f_{3}=f_{1} \cdot(D / d)\left\{1-(d / D)^{2} \cos ^{2} \theta\right\} / 2=52.9 \mathrm{~Hz}$

(4) The repetition frequency of flaws on the inner ring: $f_{\mathrm{i}}=Z \cdot\left(f_{1}-f_{2}\right)=132 \mathrm{~Hz}$

(5) The repetition frequency of flaws on the outer ring: $f_{0}=Z \cdot f_{2}=77.6 \mathrm{~Hz}$

(6) The repetition frequency of flaws on the ball: $f_{\mathrm{b}}=2 \cdot f_{3}=106 \mathrm{~Hz}$ 


\section{H. KANAI et al: : DETECTION OF FLAWS IN BALL BEARINGS}

ring and the shape factors of all parts. Table 1 shows the equations which represent the repetition frequency of the impact caused by flaws, and the frequencies calculated by the equations from the rolling speed and the shape factors of the bearingtype used in our experiments.

The resultant signal $x(n)$ is expressed as follows:

$$
x(n)=x_{1}(n) * \sum_{k=-N}^{N} \delta(n-k T)+n(n),
$$

where

$$
\delta(n)= \begin{cases}1 & (n=0) \\ 0 & (n \neq 0),\end{cases}
$$

$n(n)$ is noise, and $*$ represents the convolution.

Using Eq. (2), the Fourier transform of the vibration signal $x(n)$ is expressed as follows:

$$
X(p)=\left\{\begin{array}{c}
(2 N+1) \cdot X_{1}(p)+X_{n}(p), \\
p T=0, \pm 1, \pm 2, \cdots . \\
X_{1}(p) \frac{\sin \{2 \pi(2 N+1) p T / 2\}}{\sin (2 \pi p T / 2)}+X_{n}(p), \\
\text { otherwise, }
\end{array}\right.
$$

where $X_{1}(p)$ is the spectrum of the vibration $x_{1}(n)$, and $X_{n}(p)$ is the spectrum of the noise $n(n)$. The power spectrum of the vibration signal $x(n)$ shows many line peaks at the frequencies $f=1 / T, 2 / T, 3 / T$, ... We can observe the peaks clearly around the resonant frequency where the higher signal-to-noise ratio is obtained. Whether or not there is a flaw can be detected by the magnitude of the peaks in the power spectrum $X(p)$. Flaws are classified by the magnitudes and frequencies of those peaks.

To measure the repetition period more precisely, the method of using the cepstrum technique has been proposed. However, the method does not work as well as might be expected. Figure 2 shows the power spectra and the cepstra.

The power spectra are calculated by averaging 32 periodgrams which are computed after cutting out the vibration signal by use of a Hanning window with a length of 2,048 points. There is little difference between the power spectrum of a normal bearing: 2 (a-1) and others having flaws: 2 (a-2)-2 (a-5). It is difficult to find the frequency band in which the components of the vibration due to flaws are superior to the background noise.

The cepstra are calculated from the signal bandlimited between $1 \mathrm{kHz}$ and $9 \mathrm{kHz}$ to eliminate the effect of lower frequency components of the vibra-

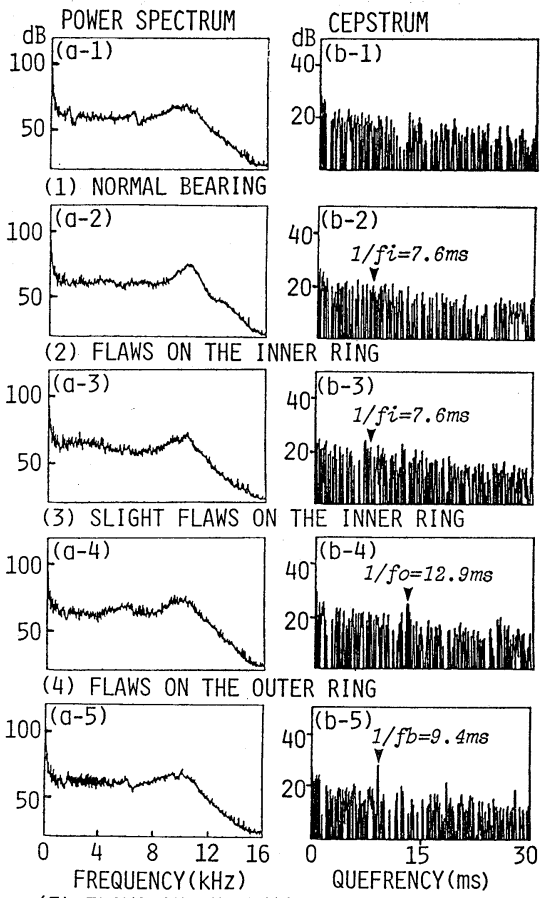

(5) FLAWS ON THE BALL

Fig. 2 An analysis of several kinds of vibration signals using the power spectral technique and the cepstrum technique.

tion which is generated when the ball passes just under the pick-up. Except for Figs. 2(b-4) and 2 (b-5), it is also difficult even by the cepstrum technique to recognize a peak at a position corresponding to the periodic interval $T$ of each flaw, as shown in Figs. 2 (b-2) and 2 (b-3). The reasons why the cepstrum does not show a peak are considered as follows:

(A) since the vibration decreases too rapidly in comparison with the repetition interval of flaws, the power of the vibration due to flaws is not larger than that of noise;

(B) the period of the vibration due to flaw pulses is irregular. That is, since the diameter of the ball is a little smaller than the diameter of the hole in the cage, there is some amount of scattering in the repetition intervals of the flaw pulses generated by many balls. Figure 3 shows an example of the distribution of repetition intervals. The repetition intervals are those at which the short-time auto-correlation functions take the maximum values near the theoretically calculated interval of a vibration due 


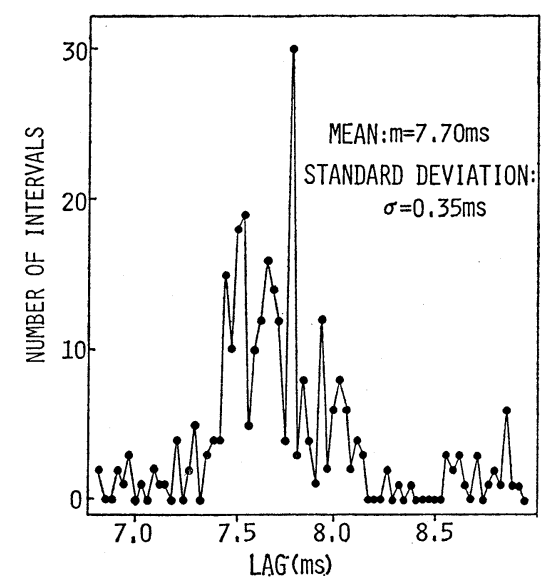

Fig. 3 The distribution of the repetition intervals of the vibration due to flaws on the inner ring. The bearing sample analyzed here is the same one as analyzed in Figs. 2 (a-2) and 2 (b-2). to flaws on the inner ring (see Table 1). The autocorrelation functions are calculated for a vibration signal band-limited between $1 \mathrm{kHz}$ and $9 \mathrm{kHz}$. In this figure, the average value $m$ of the distribution is the same as the theoretical repetition interval shown in Table 1. The standard deviation $\sigma$ takes a rather large value, and the ratio of $\sigma$ to the sampling period $T_{\mathrm{s}}(=30 \mu \mathrm{s})$ is about 11.7.

Next, we show by computer simulation the effect, both on the power spectrum and the cepstrum, of irregularity in the repetition interval of flaw pulses. Figure 4(a-1) shows the periodic exponentiallydecaying sinusoidal series which is expressed as follows:

$$
x(n)=x_{1}(n) * \sum_{k=-N}^{N} \delta(n-k T) .
$$

Figure 4(b-1) is the same signal as Fig. 4 (a-1), except for the condition where a gaussian noise $n(n)$ is
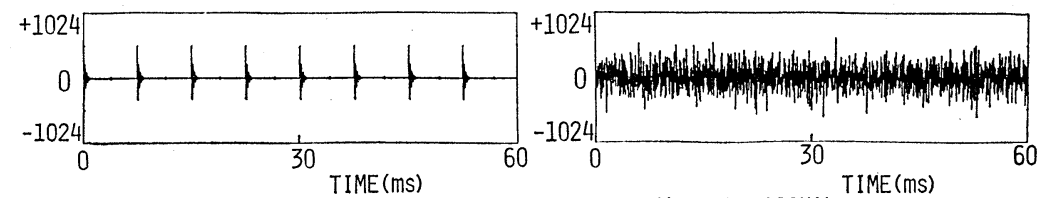

(a-1) INPUT SIGNAL

(b-1) INPUT SIGNAL
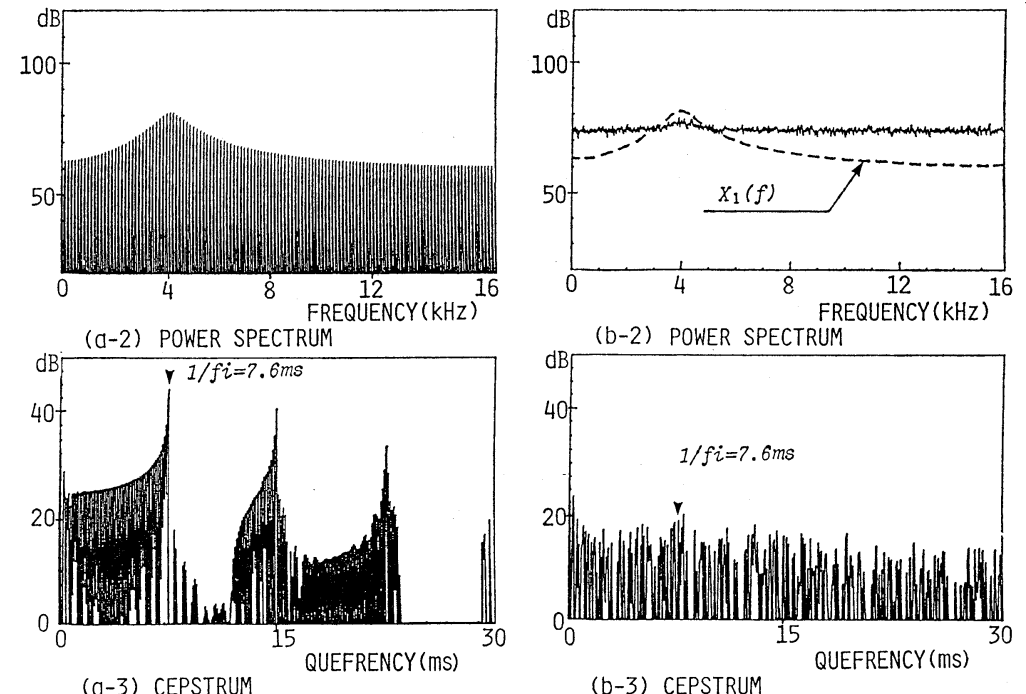

Fig. 4 The effect of the irregularity in the repetition intervals of the flaw pulses on the power spectrum and the cepstrum. (a-1): the periodic exponentially-decaying sinusoidal wave, (b-1): the periodic exponentially-decaying sinusoidal wave with irregular repetition intervals and additive gauss random noise, $X_{1}(f)$ : the envelope of the power spectrum of the resonant vibration signal without irregularity in the repetition intervals and without noise. 


\section{H. KANAI et al.: DETECTION OF FLAWS IN BALL BEARINGS}

added to the signal and there is some fluctuation in the repetition interval. The signal in Fig. $4(\mathrm{~b}-1)$ is expressed as follows:

$$
\begin{aligned}
x(n) & =x_{1}(n) * r(n)+n(n) \\
& =x_{1}(n) * \sum_{k=-N}^{N} \delta\left(n-k T-\Delta_{k}\right)+n(n),
\end{aligned}
$$

where $r(n)$ is the impulse train and $\Delta_{k}$ represents the lag for the $k$-th impulse from the expected time $k T$. The repetition interval $T+\Delta_{k}$ for the $k$-th impulse is set so that the normal probability density function $p\left(\Delta_{k} ; x\right)=N\left(m, \sigma^{2} ; x\right)$ is the same as the measured one in Fig. 3 concerning the mean $m$ and the standard deviation $\sigma$.

Figures $4(\mathrm{a}-2)$ and $4(\mathrm{a}-3)$ are the power spectrum and the cepstrum of the input signal shown in Fig. 4 (a-1), respectively. The peaks due to the repetition interval are clearly observed at the expected position in Fig. $4(\mathrm{a}-3)$. Figures $4(\mathrm{~b}-2)$ and $4(\mathrm{~b}-3)$ are the power spectrum and the cepstrum of the input signal shown in Fig. 4 (b-1), respectively. $X_{1}(f)$ in Fig. 4 (b-2) is the envelope of the power spectrum of the resonant vibration signal.

Next, the reason why clear peaks are not observed in the power spectrum $4(\mathrm{~b}-2)$ nor in the cepstrum 4 (b-3) will be explained theoretically. If a vibration has fluctuations in the periodical interval, the vibration signal is represented by Eq. (5). We assume that the distribution $\left\{\Delta_{k}\right\}$ is represented by the normal probability density function $p\left(\Delta_{k} ; x\right)=N\left(0, \sigma^{2}\right.$; $x$ ) as follows:

$$
\begin{aligned}
p\left(\Delta_{k} ; x\right) & =N\left(0, \sigma^{2} ; x\right) \\
& =\frac{1}{\sqrt{2 \pi \sigma^{2}}} \exp \left(-\frac{x^{2}}{2 \sigma^{2}}\right),
\end{aligned}
$$

where the mean value $m$ is zero and the standard deviation is $\sigma$. From other experiments we found that the standard deviation $\sigma$ was sufficiently smaller than the period $T$. Moreover, the length of the response $x_{1}(n)$ due to flaws is less than the interval $T$. Therefore, the power spectrum $|X(p)|^{2}$ of the signal $x(n)$ is calculated from Eq. (5) by the following equation, since there is no correlation between $x(n)$ and $n(n)$ :

$$
|X(p)|^{2}=\left|X_{1}(p)\right|^{2} \cdot|R(p)|^{2}+|N(p)|^{2},
$$

where $X_{1}(p), R(p)$ and $N(p)$ are the spectrum of $x_{1}(n), r(n)$ and $n(n)$, respectively.

Here, the characteristic of $|R(p)|^{2}$ is examined as follows: the auto-correlation function $C(\tau)$ of the impulse train $r(n)$ is introduced as:

$$
C(\tau)=\lim _{T_{0} \rightarrow \infty} \frac{1}{T_{0}} \int_{-T_{0} / 2}^{T_{0} / 2} r(n) r(n+\tau) d n .
$$

Then, for the interval $m T-T / 2<n \leq m T+T / 2, m=0$, $\pm 1, \pm 2, \cdots$, the product $r(n) r(n+\tau)$ is equal to 1 at only one position, and the product is equal to zero elsewhere. Thus, for the case $-T / 2<\tau \leq T / 2, C(\tau)$ is described using the delta function $\delta(n)$ as follows:

$$
\begin{aligned}
C(\tau) & =\lim _{M \rightarrow \infty} \frac{1}{M T} \sum_{m=-M / 2}^{M / 2-1} \int_{m T-T / 2}^{m T+T / 2} r(n) r(n+\tau) d n \\
& =\delta(\tau) / T, \quad-T / 2<\tau \leq T / 2 .
\end{aligned}
$$

Next, for the interval $q T-T / 2<\tau \leq q T+T / 2, C(\tau)$ is described using the probability density functions $p\left(\Delta_{k} ; x\right)$ and $p\left(\Delta_{k+q} ; y\right)$ as follows:

$$
\begin{gathered}
C(\tau)=\frac{1}{T} \int_{-\infty}^{+\infty} \int_{-\infty}^{+\infty} \delta\left(\Delta_{k}-\left(\Delta_{k+q}-\tau^{\prime}\right)\right) \\
\cdot p\left(\Delta_{k} ; x\right) p\left(\Delta_{k+q} ; y\right) d x d y,
\end{gathered}
$$

where $\tau^{\prime}=\tau-q T$. By substituting $p\left(\Delta_{k} ; x\right)$ and $p\left(\Delta_{k+q} ; y\right)$ defined in Eq. (6) into Eq. (10), the estimate of $C(\tau)$ is calculated as follows:

$$
\begin{aligned}
C(\tau)= & \frac{1}{T} \int_{-\infty}^{+\infty} \int_{-\infty}^{+\infty} \delta\left(x-\left(y-\tau^{\prime}\right)\right) \frac{1}{\sqrt{2 \pi \sigma^{2}}} \\
& \cdot \exp \left(-\frac{x^{2}}{2 \sigma^{2}}\right) \frac{1}{\sqrt{2 \pi \sigma^{2}}} \exp \left(-\frac{y^{2}}{2 \sigma^{2}}\right) d x d y \\
= & \frac{1}{T} \cdot \frac{1}{2 \pi \sigma^{2}} \int_{-\infty}^{+\infty} \exp \left(-\frac{x^{2}}{2 \sigma^{2}}\right) \\
& \cdot \exp \left(-\frac{\left(x+\tau^{\prime}\right)^{2}}{2 \sigma^{2}}\right) d x \\
= & \frac{1}{T} \cdot \frac{1}{2 \pi \sigma^{2}} \cdot \exp \left(-\frac{\tau^{\prime 2}}{4 \sigma^{2}}\right) \\
& \cdot \int_{-\infty}^{+\infty} \exp \left(-\frac{2\left(x+\tau^{\prime} / 2\right)^{2}}{2 \sigma^{2}}\right) d x .
\end{aligned}
$$

Let $y=\sqrt{2}\left(x+\tau^{\prime} / 2\right), C(\tau)$ is calculated using the relation:

$$
\frac{1}{\sqrt{2 \pi \sigma^{2}}} \int_{-\infty}^{+\infty} \exp \left(-\frac{v^{2}}{2 \sigma^{2}}\right) d v=1,
$$

as follows:

$$
\begin{aligned}
C(\tau)= & \frac{1}{T} \cdot \frac{1}{2 \sqrt{2 \pi \sigma^{2}}} \exp \left(-\frac{\tau^{\prime 2}}{4 \sigma^{2}}\right) \\
& \cdot \int_{-\infty}^{+\infty} \exp \left(-\frac{v^{2}}{2 \sigma^{2}}\right) d v \\
= & \frac{1}{T} \cdot \frac{1}{\sqrt{2 \pi(\sqrt{2} \sigma)^{2}}} \exp \left(-\frac{\tau^{\prime 2}}{2(\sqrt{2} \sigma)^{2}}\right) .
\end{aligned}
$$


Since $\delta(\tau)$ of Eq. (9) is zero except for the case $\tau=0$, and $\exp \left(-\tau^{\prime 2} /\left(2(\sqrt{2} \sigma)^{2}\right)\right)$ is sufficiently small in the case that $\left|\tau^{\prime}\right|>T / 2$, the auto-correlation function $C(\tau)$ of the impulse train $r(n)$ is approximately expressed as follows:

$$
\begin{aligned}
C(\tau)= & \frac{1}{T} \cdot \delta(\tau)+\frac{1}{T} \sum_{\substack{q=-N \\
(q \neq 0)}}^{N} \frac{1}{\sqrt{2 \pi(\sqrt{2} \sigma)^{2}}} \\
& \cdot \exp \left(-\frac{(\tau-q T)^{2}}{2(\sqrt{2} \sigma)^{2}}\right) \cdot
\end{aligned}
$$

$C(\tau)$ is expressed as the sum of two components, one of which is the periodic component $[C(\tau)]_{p}$ and the other of which is the nonperiodic components $[C(\tau)]_{\mathrm{r}}$ as follows:

$$
C(\tau)=[C(\tau)]_{\mathrm{p}}+[C(\tau)]_{\mathrm{r}},
$$

where

$$
[C(\tau)]_{\mathrm{p}}=\frac{1}{T} \sum_{q=-N}^{N} \frac{1}{\sqrt{2 \pi(\sqrt{2} \sigma)^{2}}} \exp \left(-\frac{(\tau-q T)^{2}}{2(\sqrt{2} \sigma)^{2}}\right)
$$

and

$$
\begin{aligned}
{[C(\tau)]_{\mathrm{r}}=} & \frac{1}{T} \cdot \delta(\tau)-\frac{1}{T} \cdot \frac{1}{\sqrt{2 \pi(\sqrt{2} \sigma)^{2}}} \\
& \cdot \exp \left(-\frac{\tau^{2}}{2(\sqrt{2} \sigma)^{2}}\right) \cdot
\end{aligned}
$$

Based on the Wiener-Khintchine theorem, the power spectrum $|R(p)|^{2}$ of the impulse train $r(n)$ is equal to the Fourier transform of auto-correlation function $C(\tau)$. Let $[P(p)]_{\mathrm{p}}$ and $[P(p)]_{\mathrm{r}}$ be the Fourier transforms of $[C(\tau)]_{\mathrm{p}}$ and $[C(\tau)]_{\mathrm{r}}$, respectively. Since $[C(\tau)]_{\mathrm{p}}$ is expressed as the sum of the normal probability density function $p\left(\Delta_{k} ; \tau-q T\right)=N\left(0, \sigma^{2} ; \tau-\right.$ $q T)$, the $q$-th term of which is shifted by $q T$ from the lag origin $\tau=0,[P(p)]_{\mathrm{p}}$ is expressed as follows:

$$
[P(p)]_{\mathrm{p}}=\frac{1}{T} F_{\text {norm }}(p) \sum_{q=-N}^{N} \exp (-j 2 \pi p q T),
$$

where $F_{\text {norm }}(p)$ is the Fourier transform of the normal probability density function $N\left(0,2 \sigma^{2} ; n\right)$ as follows:

$$
\begin{aligned}
F_{\text {norm }}(p) & =\boldsymbol{F}\left[N\left(0,2 \sigma^{2} ; n\right)\right] \\
& =\exp \left(-2(\pi \sqrt{2} \sigma p)^{2}\right) .
\end{aligned}
$$

The second factor in Eq. (16) is expressed using the formula for geometrical series as follows:

$$
\begin{aligned}
& \sum_{q=-N}^{N} \exp (-j 2 \pi p q T) \\
& = \begin{cases}(2 N+1), & p T=0, \pm 1, \pm 2, \cdots \\
\frac{\sin \{2 \pi(2 N+1) p T / 2\}}{\sin (2 \pi \cdot p T / 2)}, & \text { otherwise. }\end{cases}
\end{aligned}
$$

Since $N$ is sufficiently large, we can assume that Eq. (18) shows line peaks at $p T=0, \pm 1, \pm 2, \cdots$. On the other hand, the power spectrum of the non-periodic components is expressed as

$$
[P(p)]_{\mathrm{r}}=\frac{1}{T}\left\{1-F_{\text {norm }}(p)\right\},
$$

where 1 is the Fourier transform of the delta function $\delta(n)$. From Eqs. (18) and (19), $|R(p)|^{2}$ is expressed as

$$
|R(p)|^{2}=\left\{\begin{array}{c}
\frac{1}{T}\left\{F_{\text {norm }}(p) \cdot(2 N+1)+1-F_{\text {norm }}(p)\right\}, \\
p T=0, \pm 1, \pm 2, \cdots . \\
\frac{1}{T}\left\{F_{\text {norm }}(p) \cdot \frac{\sin \{2 \pi(2 N+1) p T / 2\}}{\sin (2 \pi \cdot p T / 2)}\right. \\
\left.\quad+1-F_{\text {norm }}(p)\right\}, \text { otherwise. }
\end{array}\right.
$$

Figure $5(\mathrm{~b})$ shows the power spectrum directly calculated by Eq. (20). Figure 5 (a) shows the power spectrum of the computer-synthesized impulse train $r(n)$ with random intervals, which is defined in Eq. (5) in the case in which the noise $n(n)=0$. The two power spectra almost coincide with each other. The first term of Eq. (20) shows line peaks clearly at the frequencies $f=n / T, n=1,2, \cdots$, mainly in the low frequency range. Since the peakes are observed clearly in the case that $[P(p)]_{\mathrm{p}}>[P(p)]_{\mathrm{r}}$, the upper limit frequency $f_{c}$, up to which we can observe the peaks, is expressed as:

$$
(2 N+1) \cdot F_{\text {norm }}\left(f_{\mathrm{c}}\right)=1-F_{\text {norm }}\left(f_{\mathrm{c}}\right) .
$$

From Eq. (21), $f_{\mathrm{c}} / f_{\mathrm{s}}$ is expressed as follows:

$$
f_{\mathrm{c}} / f_{\mathrm{s}}=\frac{1}{2 \pi} \sqrt{\ln N_{\mathrm{r}}} \cdot \frac{1}{\sigma / T_{\mathrm{s}}},
$$

where $N_{\mathrm{r}}=(2 N+2)$, and $f_{\mathrm{s}}=1 / T_{\mathrm{s}}$ denotes the sampling frequency $(=33 \mathrm{kHz}$, see Fig. 1). Figure 5 (c) shows the relation between the standard deviation of the lag $\left\{\Delta_{k}\right\}$ and the upper limit frequency $f_{\mathrm{c}}$.

The power spectrum $|X(p)|^{2}$ of $x(n)$ is the product of $|R(p)|^{2}$ and the power spectrum $\left|X_{1}(p)\right|^{2}$ of the vibration $x_{1}(n)$ due to flaws superimposed by noise power spectrum $|N(p)|^{2}$, as shown in Eq. (7). Therefore, it is hard to detect the line spectra in the power spectrum $|X(p)|^{2}$ in the case in which vibrations due to flaws have fluctuations in the periodical interval and the power of the vibration due to flaws is not superior to that of the noise except for the limited high frequency region. We cannot detect and classify flaws by these proposed automatic methods in such cases. 


\section{H. KANAI et al.: DETECTION OF FLAWS IN BALL BEARINGS}
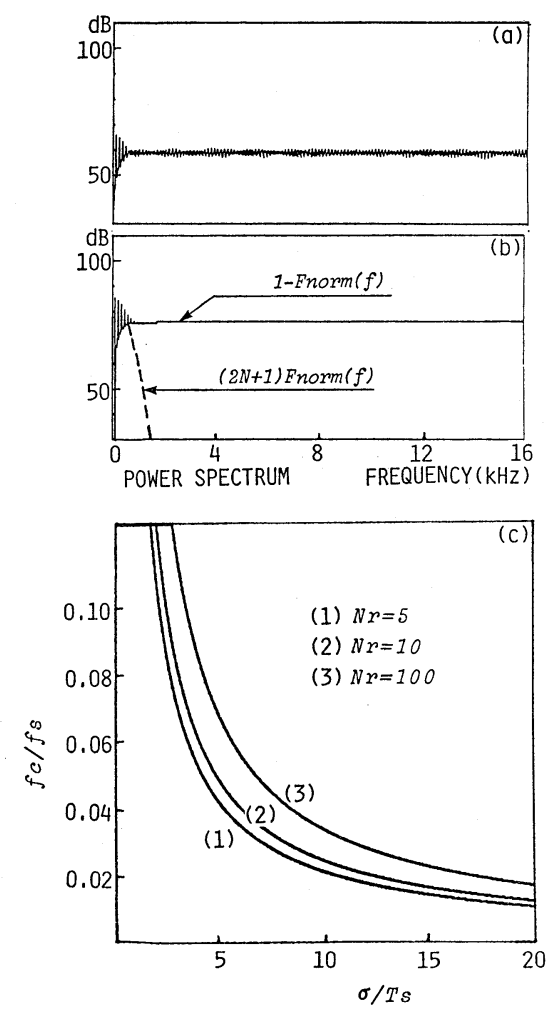

Fig. 5 The effect of the irregularity in the repetition intervals of the periodic impulse train on the power spectrum. (a): the power spectrum of the computer-synthesized impulse train with random intervals, (b): the power spectrum directly calculated by Eq. (20), (c): relation between the standard deviation $\sigma$ and the normalized upper limit frequency $f_{\mathrm{c}} / f_{\mathrm{s}}$ to detect the line peaks. $f_{\mathrm{s}}=1 / T_{\mathrm{s}}$ : the sampling frequency, $N_{\mathrm{r}}=(2 N+2), N$ : number of intervals.

\section{FLAW DETECTION BASED ON THE ENVELOPE SIGNAL OF THE SQUARED MAGNITUDE OF THE NARROW BAND SIGNAL}

We propose a new, reliable method of detecting vibration due to flaws. The input vibrational signal is band-limited, using a short-time (16 point) Fourier transform, and 8 narrow band-passed signals are obtained. Then, the significant frequency band, by which the best detection of flaws is achieved, is selected from the 8 narrow bands. The envelope signal is obtained by squaring the narrow bandpassed signal and passing it though a low-pass filter. The periodicity is detected from the envelope signal. The details of this processing are explained hereafter.

First, a band-limited signal $y(n), n=[-N, N-1]$ is made from the $2 N$ point input data sequence $x(n)$, $n=[-N, N-1]$, where the duration $2 N$ is sufficiently shorter than the period of the rotation of the inner ring. In our experiments the duration $2 N$ is set at 16. Then, at most only one pulse is observed in the duration. The spectrum $Y(p)$ of the band-limited signal $y(n)$ is represented using Fourier transform $X(p)$ of $x(n)$ and the transfer function $W(p)$ of a narrow band-pass filter as follows:

$$
Y(p)=W(p) \cdot X(p) .
$$

Second, the squared signal $z(n)$ of the bandpassed signal $y(n)$ is calculated. Using the relation $\left.\boldsymbol{F}\left[y^{*}(n)\right]=Y^{*}(-p),{ }^{3}\right)$ where $\boldsymbol{F}[]$ denotes the Fourier transform and $y^{*}(n)$ and $Y^{*}(-p)$ denote the complex conjugates of $y(n)$ and $Y(-p)$, respectively, the spectrum $Z(p)$ of the squared band-passed signal $z(n)=|y(n)|^{2}$ is represented as follows:

$$
\begin{aligned}
Z(p) & =\boldsymbol{F}\left[y(n) \cdot y^{*}(n)\right] \\
& =\boldsymbol{F}[y(n)] * \boldsymbol{F}\left[y^{*}(n)\right] \\
& =Y(p) * Y^{*}(-p) \\
& =\sum_{k=-N}^{N-1} Y(k) Y^{*}(k-p) \\
& =\sum_{k=-N}^{N-1} W(k) X(k) W^{*}(k-p) X^{*}(k-p) .
\end{aligned}
$$

Since $z(n)$ is the squared signal, the spectrum $Z(p)$ has low frequency components. From Eq. (2), the spectrum $X(p)$ is the sum of two components: the resonant vibration $S(p)$ and a random noise $N(p)$, as follows:

$$
X(p)=S(p)+N(p) .
$$

Using Eqs. (24) and (25), $Z(p)$ is represented as follows:

$$
\begin{aligned}
Z(p)= & \sum_{k=-N}^{N-1} W(k) W^{*}(k-p)\left\{S(k) S^{*}(k-p)\right. \\
& +S(k) N^{*}(k-p)+N(k) S^{*}(k-p) \\
& \left.+N(k) N^{*}(k-p)\right\} .
\end{aligned}
$$

In Eq. (26) the components due to the second term $S(k) N^{*}(k-p)$ and the third term $N(k) S^{*}(k-p)$ take small values since there is no correlation between 
$S(k)$ and $N(k)$. From other experiments, we found the fact that the resonant signal $s(n)$ due to flaws has a broad-phase characteristic in the frequency domain, while the noise $n(n)$ has a random-phase characteristic. Therefore, the components due to the fourth term $N(k) N^{*}(k-p)$ take a smaller value than that due to the first term $S(k) S^{*}(k-p)$. That way, the method of calculating the band-passed signal has the advantage of emphasizing the resonant vibration components buried in the random noise.

The reasoning can be explained by computer simulation as follows: Figs. 6 (a-1) and 6 (a-2) show the computer-synthesized signals for the resonant vibration signal with a broad phase, as shown in Fig. 6 (c-1), and for a noise with a random phase, as shown in Fig. 6 (c-2), respectively. In these figures, the total length of the data $x(n)$ is 2,048 points and the value of the damping factor $\alpha=1 / 10$. This value is almost the same as that of the resonant vibration due to flaws. In Eq. (26), the component due to the first term has a larger value than that due to the fourth term, even if $S(p)$ and $N(p)$ have the same power spectra as shown in Figs. 6 (b-1) and 6 (b-2). Figures $6(\mathrm{~d}-1)$ and 6 (d-2) show the analyzed results of the two signals shown in Figs. 6 (a-1) and 6 (a-2), using our method. As shown in Figs. 6 (d-1) and 6 (d-2), the power spectrum of the envelope of the narrow, band-passed signal with a uniform phase is about $17 \mathrm{~dB}$ larger than that of the signal with a random phase. In this figure the band-passed signal $y(n)$ is calculated using a $2 N=16$ points Fast Fourier Transform (FFT). The amount of noise attenuation is changed by the length $2 N$ of the data in Eq. (26). Figure 6 (e) shows the relation between the amount of noise attenuation and the length of the data. The amount of noise attenuation increases by decreasing the length $2 N$ of the data, or when the value of the damping factor $\alpha$ is larger because of the following two reasons: (1) the width of the main-lobe of the narrow, band-pass filter increases by decreasing the length $2 N$ of the data, and (2) the phase characteristic of $x(n)$ is broader when $\alpha$ takes a larger value. However, when we increase the width of the main-lobe too much, the power of the components other than the resonant vibration increase, and the signal-tonoise ratio decreases. Thus, we use a time window with a length of 16 points.

The method of calculating the squared signal has another advantage; it deletes the influence of fluctuations of the period, as explained below: from other
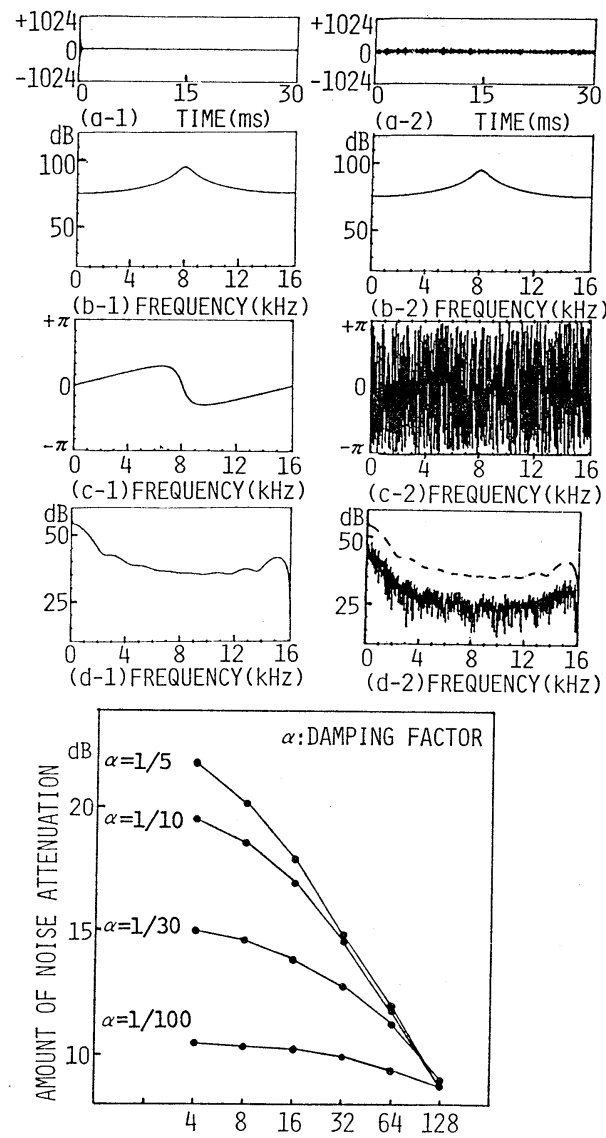

(e) LENGTH OF THE WINDOW IN FFT

Fig. 6 Improvement of the signal to noise ratio by the proposed method. (a-1): computer-synthesized resonant vibration signal with an almost constant phase, (a-2): computer-synthesized noise with a random phase, (b-1) and (c-1): the power spectrum and the phase of the signal shown in (a-1), (b-2) and (c-2): the power spectrum and the phase of the signal shown in (a-2), (d-1) and (d-2): the results obtained by the proposed method from the signals (a-1) and (a-2), respectively, (e): relation between the amount of noise attenuation and the length of the data.

investigations, the response $x_{1}(n)$ shown in Eq. (5) to one flaw and the duration $2 N$ of the time window $w(n)$ are sufficiently shorter than the period of the repetition interval $T$ of flaws, and there is no correlation between $x_{1}(n)$ and $n(n)$. Therefore, the 


\section{H. KANAI et al.: DETECTION OF FLAWS IN BALL BEARINGS}

squared signal $z(n)$ is expressed as follows:

$$
\begin{aligned}
z(n)= & |y(n)|^{2} \\
\simeq & \left|x_{1}(n) * w(n)\right|^{2} * \sum_{k=-N}^{N} \delta\left(n-k T-\Delta_{k}\right) \\
& +|n(n) * w(n)|^{2} .
\end{aligned}
$$

Equation (27) is the same as Eq. (5) except for the data window, that is, $\left|x_{1}(n) * w(n)\right|^{2}$ and $|n(n) * w(n)|^{2}$ are used instead of $x_{1}(n)$ and $n(n)$, respectively. Except for the D.C. components, there is no correlation between $\left|x_{1}(n) * w(n)\right|^{2}$ and $|n(n) * w(n)|^{2}$. Because of the above two reasons, the power spectrum $|Z(p)|^{2}$ of the squared signal $z(n)$ is expressed as follows:

$$
|Z(p)|^{2}=\left|X_{1}^{\prime}(p)\right|^{2} \cdot|R(p)|^{2}+\left|N^{\prime}(p)\right|^{2},
$$

where $\left|X_{1}{ }^{\prime}(p)\right|^{2}$ is the power spectrum of $\left|x_{1}(n) * w(n)\right|^{2}$ and $\left|N^{\prime}(p)\right|^{2}$ is that of the sequence $|n(n) * w(n)|^{2}$. Even if the original signal $x_{1}(n)$ does not contain low frequency components, the low frequency components of $\left|X_{1}{ }^{\prime}(p)\right|^{2}$ are large in magnitude. Therefore, $|Z(p)|^{2}$ shows clear peaks in low frequencies in spite of the effect of $|R(p)|^{2}$ shown in Eq. (20) and Fig. 5 which represent the irregularity of the repetition intervals.

Figure 7 shows the result of the computer-syn-

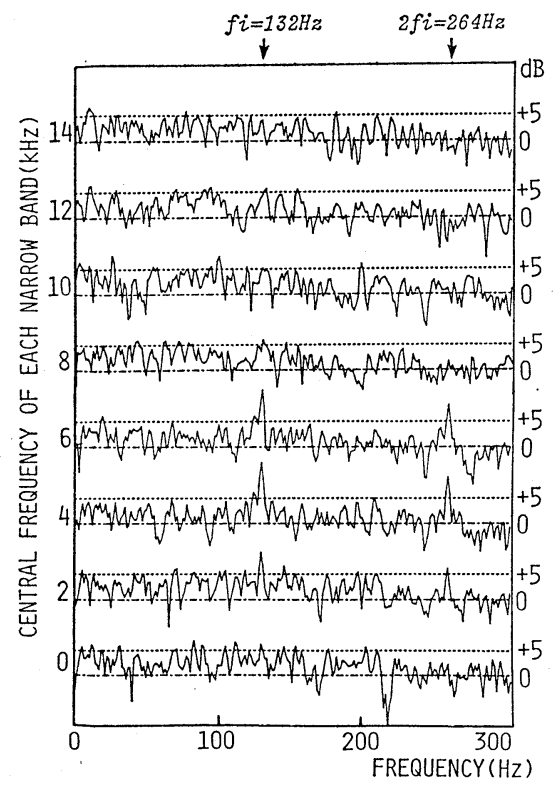

Fig. 7 The results obtained by the proposed method from the computer-synthesized signal shown in Fig. 4 (b-1). thesized signal shown in Fig. 4 (b-1), which is the repetitive exponentially-decaying sinusoidal signal with additive gauss random noise. The period of this wave cannot be detected by a conventional period detection methods, such as the cepstrum technique as shown in Fig. 4 (b-3). It is, however, possible to detect its period by our method at $2 \mathrm{kHz}$, $4 \mathrm{kHz}$ and $6 \mathrm{kHz}$, which are the frequency bands near the resonant frequency, as shown in Fig. 7. These results from computer simulations indicate that this method is suitable as a diagnostic technique.

\section{EXPERIMENTS AND THE RESULTS}

The process of the flaw detection method proposed here is explained at this time. At first, the vibration signal is divided into many segments by multiplying short time (16 point) Hanning windows. Adjacent segments overlap each other by a half-length of the segment. The vibration signal is band-limited by calculating Fourier transforms for all the segments. Each band-limited signal is squared and movingaveraged using a 16 point Hanning window so that only low frequency components due to flaws are extracted. Then, a envelope signal is generated by picking up one sample every third segment, and the period of the flaws is estimated by the Fourier Transform of the envelope signal (1,024 points, Hanning window, averaged by summing up them 7 times).

The experimental results obtained by the proposed method are shown in Fig. 8. Figures 8 (2) and 8 (3) show the results for the bearings having flaws on the inner ring. The vibration signals used here are the same as those used to obtain Figs. 2 (2) and 2 (3). Especially in the $4 \mathrm{kHz}$ band, peaks appear clearly at the frequencies: $f_{1}$ and $2 f_{\mathrm{i}}$, which just coincide with the theoretical ones. On the other hand, these peaks cannot be observed by other proposed automatic methods as shown in Figs. 2(2) and 2(3). Figure 8 (1) shows the results with a normal bearing. Clear peaks cannot be observed in the $4 \mathrm{kHz}$ band and the higher frequency bands. Figures 8 (4) and 8 (5) show the results for bearings having flaws on the outer ring and on the ball, respectively. In these figures, peaks also appear clearly at the frequencies: $f_{\mathrm{o}}$ and $f_{\mathrm{b}}$, respectively (see also Table 1 ). Therefore, the flaws can be detected and classified using the $4 \mathrm{kHz}$ band signal by checking whether or not there are peaks at the frequencies corresponding to the theoretical ones. 

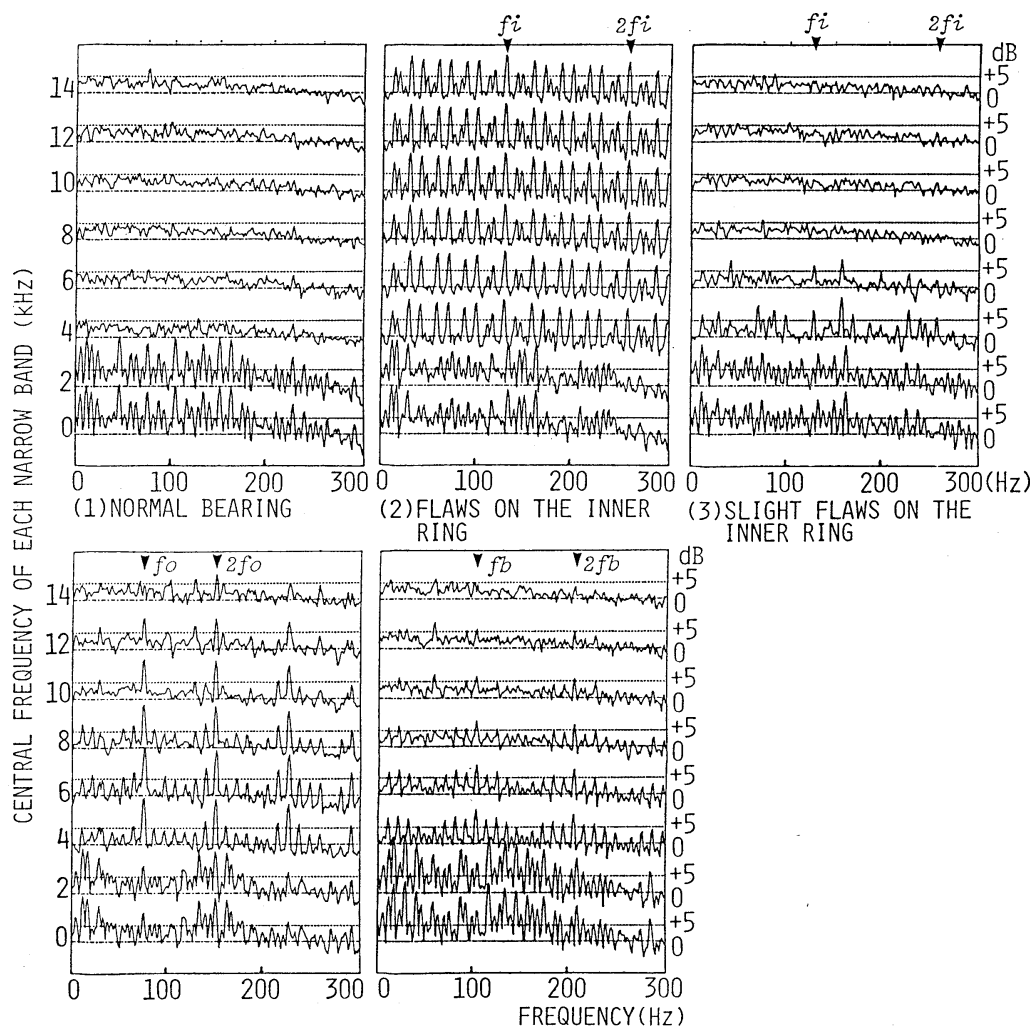

3) SLIGHT FLAWS ON THE

INNER RING

(4)FLAWS ON THE OUTER

(5) FLAWS ON THE BALL RING

Fig. 8 The experimental results obtained by the proposed method. The vibration signals used here are the same as those used in Fig. 2. The peaks at $f_{\mathrm{i}}, f_{\mathrm{o}}$ and $f_{\mathrm{b}}$ correspond to the frequencies in Table 1.

\section{CLASSIFICATION OF FLAWS AND ITS EXPERIMENTAL RESULTS}

It is easily found whether a sample is normal or has some flaws by classifying the feature vector introduced next. The components of the feature vector are the power of peaks appearing at the repetition frequencies, which are calculated theoretically for the three kinds of flaws. The kinds of flaws are also discriminated by these repetition frequencies. We used 241 samples for the classification experiments. Figure 9 shows the classification results of these samples by Bayes decision. In order to check which band envelope is the best to detect and classify flaws, feature vectors are calculated for 8 bands and the accuracy rates are computed. The components of the feature vector used in the experiments are the values of peaks appearing at the three repetition

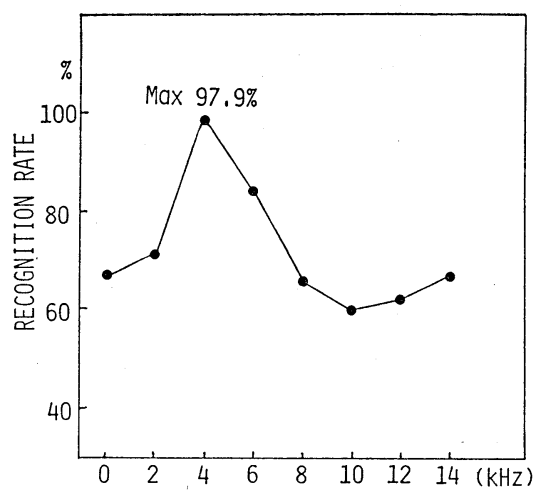

CENTRAL FREQUENCY OF EACH NARROW BAND

Fig. 9 The relation between the recognition rate and the narrow bands used in the classification process. 241 samples were used in these experiments. 


\section{H. KANAI et al.: DETECTION OF FLAWS IN BALL BEARINGS}

frequencies. The flaws were classified using the 4 $\mathrm{kHz}$ band with an accuracy rate of about $97.9 \%$ into four categories: normal, flaws on the inner ring, flaws on the outer ring and flaws on the balls. By using this feature vector, the detection and classification do not depend on the total power of the input signal.

\section{CONCLUSIONS}

A new method is proposed for the automatic detection and classification of slight flaws in ball bearings using vibrational signals. Slight flaws, which are not detected by other proposed automatic methods such as the cepstrum technique, are easily detected and classified by our new method with an accuracy rate of $97.9 \%$ into four categories; normal, flaw on the inner ring, flaw on the outer ring and flaw on the balls.

\section{REFERENCES}

1) T. Igarashi, "Sound of rolling bearings," Lubr. 22, 751-756 (1977) (in Japanese).

2) S. Braun and B. Datner, "Analysis of roller/ball bearing vibrations," Trans. Am. Soc. Mech. Eng., J. Mech. Des. 101, 118-125 (1979).

3) A. V. Oppenheim and R. W. Schafer, Digital Signal Processing (Prentice-Hall, Englewood Cliffs, 1975), p. 110, Table 3.2.

4) Y. W. Lee, Statistical Theory of Communication (John Wiley \& Sons, New York, 1960), Chap. 8. 